\title{
Establishment of reproducible osteosarcoma rat model using orthotopic implantation technique
}

\author{
ZHE YU*, HONGHUI SUN*, QINGYU FAN, HUA LONG, TONGTAO YANG and BAO'AN MA \\ Center of Orthopedic Surgery, Orthopedics Oncology Institute of Chinese PLA, Tangdu Hospital, \\ Fourth Military Medical University, Xi'an, Shaanxi 710038, P.R. China
}

Received November 26, 2008; Accepted February 18, 2009

DOI: $10.3892 / o r \_00000338$

\begin{abstract}
In experimental musculoskeletal oncology, there remains a need for animal models that can be used to assess the efficacy of new and innovative treatment methodologies for bone tumors. Rat plays a very important role in the bone field especially in the evaluation of metabolic bone diseases. The objective of this study was to develop a rat osteosarcoma model for evaluation of new surgical and molecular methods of treatment for extremity sarcoma. One hundred male SD rats weighing $125.45 \pm 8.19 \mathrm{~g}$ were divided into 5 groups and anesthetized intraperitoneally with $10 \%$ chloral hydrate. Orthotopic implantation models of rat osteosarcoma were performed by injecting directly into the SD rat femur with a needle for inoculation with SD tumor cells. In the first step of the experiment, $2 \times 10^{5}$ to $1 \times 10^{6} \mathrm{UMR} 106$ cells in $50 \mu 1$ were injected intraosseously into median or distal part of the femoral shaft and the tumor take rate was determined. The second stage consisted of determining tumor volume, correlating findings from ultrasound with findings from necropsia and determining time of survival. In the third stage, the orthotopically implanted tumors and lung nodules were resected entirely, sectioned, and then counter stained with hematoxylin and eosin for histopathologic evaluation. The tumor take rate was $100 \%$ for implants with $8 \times 10^{5}$ tumor cells or more, which was much less than the amount required for subcutaneous implantation, with a high lung metastasis rate of $93.0 \%$. Ultrasound and necropsia findings matched closely $(\mathrm{r}=0.942 ; \mathrm{p}<0.01)$, which demonstrated that Doppler ultra-
\end{abstract}

Correspondence to: Dr Bao'an Ma, Center of Orthopedic Surgery, Orthopedics Oncology Institute of Chinese PLA, Tangdu Hospital, Fourth Military Medical University, Xi'an, Shaanxi 710038, P.R. China

E-mail: yuzhe780519@yahoo.com.cn

${ }^{*}$ Contributed equally

Abbreviations: SEM, scan electron microscope; TEM, transmission electron microscope; SD, Sprague Dawley; ATCC, American Type Culture Collection; PBS, phosphate-buffered saline; HE, hematoxylineosin; PET, positron emission tomography; DIO, Doppler digital image optimizer; FMMU, Fourth Military Medical University

Key words: osteosarcoma, rat, orthotopic model, ultrasound sonography is a convenient and reliable technique for measuring cancer at any stage. Tumor growth curve showed that orthotopically implanted tumors expanded vigorously with time-lapse, especially in the first 3 weeks. The median time of survival was 38 days and surgical mortality was $0 \%$. The UMR106 cell line has strong carcinogenic capability and high lung metastasis frequency. The present rat osteosarcoma model was shown to be feasible: the take rate was high, surgical mortality was negligible and the procedure was simple to perform and easily reproduced. It may be a useful tool in the investigation of antiangiogenic and anticancer therapeutics. Ultrasound was found to be a highly accurate tool for tumor diagnosis, localization and measure-ment and may be recommended for monitoring tumor growth in this model.

\section{Introduction}

Osteosarcomas are the prominent primary bone cancers in humans, excluding hemopoietic malignancies. They mainly affect children and adolescents and are usually highly aggressive and eventually lethal. The current grading and staging systems of osteosarcoma are based on a combination of clinicopathologic features, namely, the histological appearance of the tumor and its local and/or disseminated spread (1). Although this kind of malignant tumor is one of the main causes of death and morbidity, the study of the biology of osteosarcoma has been limited due to the lack of suitable laboratory animal models. Animal models provide important knowledge on the pathophysiological conditions that lead to a given disease and allow the preclinical evaluation of new effective treatments. Research using animal models acts as the bridge between in vitro studies and human clinical trials. The mouse has become popular in skeletal research because of the ease with which its genome can be manipulated and investigated. The rat is also a widely used model, due to its availability, low cost, easy handling, easy housing and resistance (2).

The most used technique for the detection of bone tumor is X-ray examination. However, other techniques have been reported to characterize bone tumors in animal models: scintigraphy (3), microcomputed tomography-X (4), computerised tomography $(\mathrm{CT})(5,6)$ and magnetic resonance imaging (MRI) (7). Other new analytical tools are in development and adaptable to rats: the PET (positron emission tomography) can be performed with $2-\left[{ }^{18} \mathrm{~F}\right]$ fluoro- 
2-deoxy-D-glucose (FDG) or 99mTc-bisphosphonate to detect metastases (8). Doppler ultrasonography is not an emerging method, but it is highly regarded by researchers. Except for a convenient and harmless technique, ultrasound can be used to visualize and measure cancer at any stage, including the growth of the primary tumor and the assessment of angiogenesis in pathological region, even in living body (9-11).

In vivo studies have also tended in the past to rely on subcutaneous injection of tumor cells (12). Orthotopic models are far less common but essential for the true appreciation of tumor biology and characteristics. Further, there is a need for an orthotopic model in which the disease course can be readily followed in vivo, avoiding the need to cull large numbers of animals to assess and establish significance (13).

\section{Materials and methods}

Rats and cell line. Male SD rats, 4- to 5-weeks old, were purchased from the laboratory animal research centre of Fourth Military Medical University (FMMU). The rats were maintained in microisolator cages under specific pathogen-free conditions. The temperature was maintained at $24^{\circ} \mathrm{C}$ and the animals were exposed to a 24 -h circadian rhythm with free access to water and food. The study was previously approved by the FMMU Ethics Committee for Animal Research and all experiments were performed in accordance with sound ethical principles.

The UMR106 osteosarcoma cell line syngeneic to SD rats was purchased from the American Type Culture Collection (ATCC; Manassas, VA). The cell line was cultured in RPMI10\% FBS (Sigma) supplemented with L-glutamine (2 mM), penicillin $(100 \mathrm{U} / \mathrm{ml})$ and streptomycin $(100 \mu \mathrm{g} / \mathrm{ml})$ confirmed to be mycoplasma-free by routine testing.

Preparation for electron microscopy. For observation of cell morphology, UMR106 cells were attached to a plastic cover slip that had been coated with $1 \%$ poly-L-lysine in water for $15 \mathrm{~min}$. The cells were fixed with $1.5 \%$ glutaraldehyde in $0.1 \mathrm{M}$ phosphate-buffered saline (PBS), $\mathrm{pH} 7.4$, passed through an alcohol gradient, dried in a Ladd Critical Point Dryer and coated with platinum in a Plaron SEM Coating System. The fixed cells were examined with JEOL JSM-35 CF scanning electron microscope (SEM). For observation of cell intra-cellular structure, cell preparation was fixed with $1.5 \%$ glutaraldehyde in $0.1 \mathrm{M}$ cacodylate buffer, $\mathrm{pH} 7.4$, for $1 \mathrm{~h}$ at $4^{\circ} \mathrm{C}$. The specimens were washed, treated with $1 \%$ osmium tetroxide in $0.1 \mathrm{M}$ cacodylate buffer and passed through an alcohol gradient. They were further treated with propylene oxide and then embedded. The ultrathin sections were cut with an MT2 Sorvall ultramicrotome and examined with a JEOL-100-CX transmission electron microscope (TEM).

Orthotopic implantation technique. One hundred male SD rats weighing $125.45 \pm 8.19 \mathrm{~g}$ were divided into 5 groups at random: Group A $(n=20), 2 \times 10^{5}$ cells; Group B $(n=20)$, $4 \times 10^{5}$ cells; Group C $(n=20), 6 \times 10^{5}$ cells; Group D $(n=20)$, $8 \times 10^{5}$ cells; and Group E $(n=20), 1 \times 10^{6}$ cells. Then all the animals were anesthetized intraperitoneally with $10 \%$ chloral hydrate at a dosage of $0.1 \mathrm{ml} / 30 \mathrm{~g}$ live weight and the operative field was prepared with iodine and draped. Orthotopic implantation models of rat osteosarcoma were performed by injecting directly into the SD rat femur with a syringe and 25 gauge needle for inoculation with SD tumor cells. Respectively, $2 \times 10^{5}$ to $1 \times 10^{6}$ UMR106 cells in $50 \mu 1$ were injected intraosseously into median or distal part of the femoral shaft and the tumor take rate was determined.

Detection of tumor volume by ultrasound. After implantation, tumor size was determined in living body weekly by measuring the largest and the smallest diameters. Tumor volume was calculated according to the following formula recommended by Carlsson et al (14).

Tumor volume $\left(\mathrm{mm}^{3}\right)=$ largest diameter $(\mathrm{mm}) \times$ smallest diameter $(\mathrm{mm})^{2 / 2}$

Tumor measure procedures were performed with ultrasonography and tumor growth curve was made. For ultrasound imaging we used a $3.0 \mathrm{MHz}$ real-time scanner of SSA-550 (Toshiba Medical Systems, Tokyo, Japan). We used a Doppler digital image optimizer (DIO) with adaptive image processing for high sensitivity. Necropsy was conducted immediately after death of each tumor-bearing rat. Lung nodules from metastasis were identifed and orthotopically implantated tumor was removed for ultrasound imaging and histological measurement by sliding caliper. The findings from ultrasound were correlated with findings from necropsia and the ultrasound imaging confidence level was determined.

Histopathological examination. Implantation tumor and lung sections were fixed in buffered isotonic formaldehyde $(100 \mathrm{ml}$ of $37 \%$ formaldehyde solution, $900 \mathrm{ml}$ distilled water, $4 \mathrm{~g}$ monobasic sodium phosphate and $6.5 \mathrm{~g}$ dibasic sodium phosphate) and embedded in paraffin. Twenty-four hours later, samples were immersed in $70 \%$ alcohol, stained with hematoxylin-eosin (H\&E) and examined histopathologically by a pathologist in a blinded manner.

Statistical analysis. SPSS11.0 was used for data variation analysis. Tumor volumes obtained from ultrasound and necropsy and expressed as average values \pm standard error were compared using simple linear regression. Survival rates were determined with the $\chi^{2}$ test. P-values $<0.05$ were taken to indicate statistical significance.

\section{Results}

UMR106 rat osteosarcoma cell morphology. Under SEM, UMR106 osteosarcoma cells had a thick cell coat and fusiform or triangle shape. Most tumor cells grew adherently and displayed elongated close needling processes. Ditissimus mucus wrapped the cell body (Fig. 1A). Under TEM, the tumor cells histologically demonstrated features of UMR106 with large nuclei, visible nucleoli, loose chromatin and widespread atypical mitosis (Fig. 1B).

Tumor take and lung metastasis rates. UMR106 osteosarcoma cells inoculated in femur had an implantation and growth rate of $100 \%$ on groups D and E and only $5 \%$ on 
A

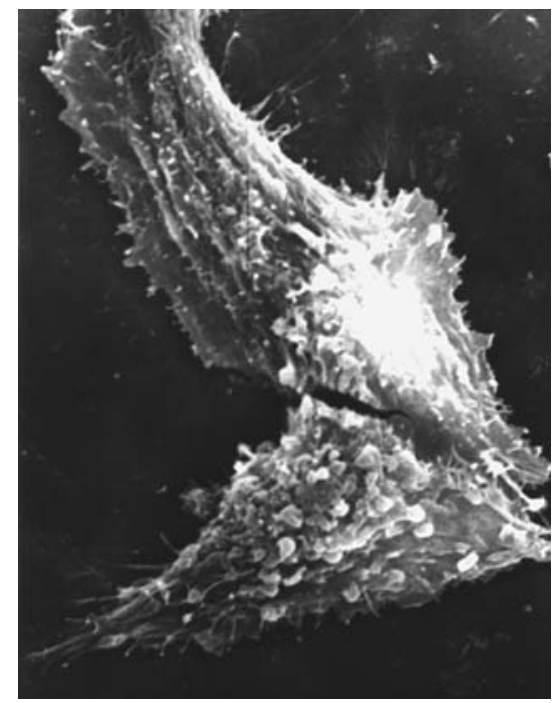

B

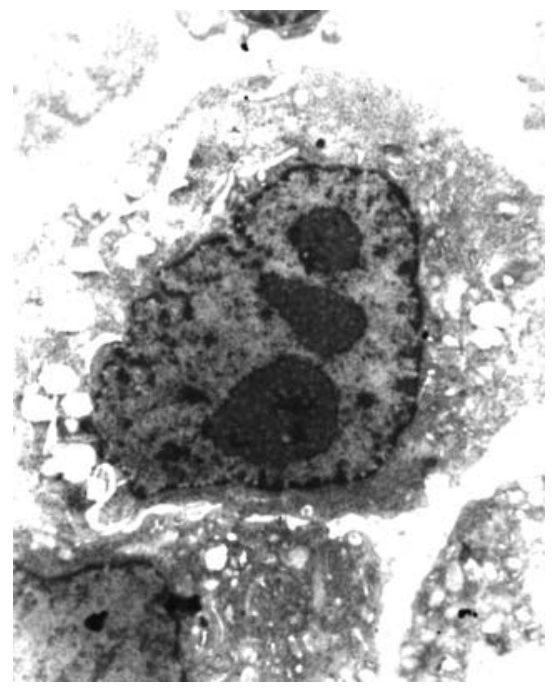

Figure 1. UMR106 osteosarcoma cells under scan (A) and transmission (B) electron microscope. (SEM x4000, TEM x6000).

Table I. Tumor take rate vs. number of cells implanted in the rat femurs.

\begin{tabular}{lccccrr}
\hline Group & $\begin{array}{c}\text { No. of tumor cells } \\
\text { implanted (cells) }\end{array}$ & $\begin{array}{c}\text { Animals } \\
\text { inoculated }\end{array}$ & $\begin{array}{c}\text { Animals with } \\
\text { tumor }\end{array}$ & $\begin{array}{c}\text { Tumor take } \\
\text { rate (\%) }\end{array}$ & $\begin{array}{c}\text { Animals with lung } \\
\text { nodules }\end{array}$ & $\begin{array}{c}\text { Lung metastasis } \\
\text { rate }(\%)\end{array}$ \\
\hline Group A & $2 \times 10^{5}$ & 20 & 1 & 5 & 0 & 0 \\
Group B & $4 \times 10^{5}$ & 20 & 4 & 20 & 60 & 10 \\
Group C & $6 \times 10^{5}$ & 20 & 12 & 100 & 19 & 50 \\
Group D & $8 \times 10^{5}$ & 20 & 20 & 100 & 20 & 100 \\
Group E & $1 \times 10^{6}$ & 20 & 20 & & & 5 \\
\hline
\end{tabular}

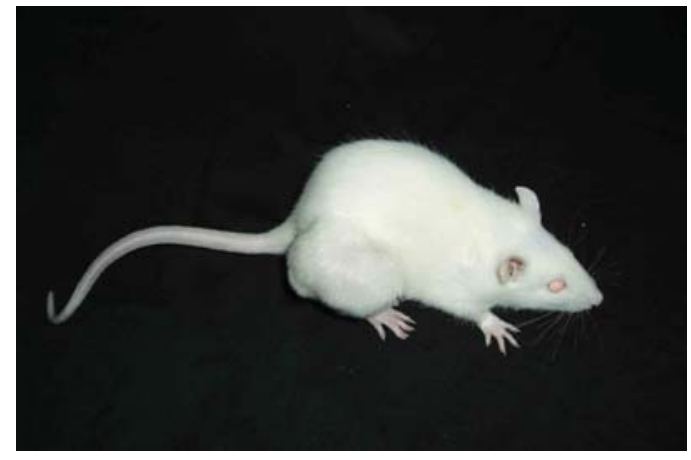

Figure 2. UMR 106 osteosarcoma cells inoculated in femur had an implantation and growth rate of $100 \%$ at $8 \times 10^{5}$ cells.

group A (Table I). The tumors in groups D and E took on expansive growth, affecting the neighbouring organs, invading into cavitas pelvis and even inducing urethra and rectum compression (Figs. 2 and 3). Doppler ultrasonography demonstrated a well-demarcated low echo mass accompanied with a central necrosis and hypervascular property of the tumor. Thus, the formed UMR106 osteosarcoma was pathologically classified into a poorly differentiated bone cancer demarcated with surrounding bony and loose connective tissue.

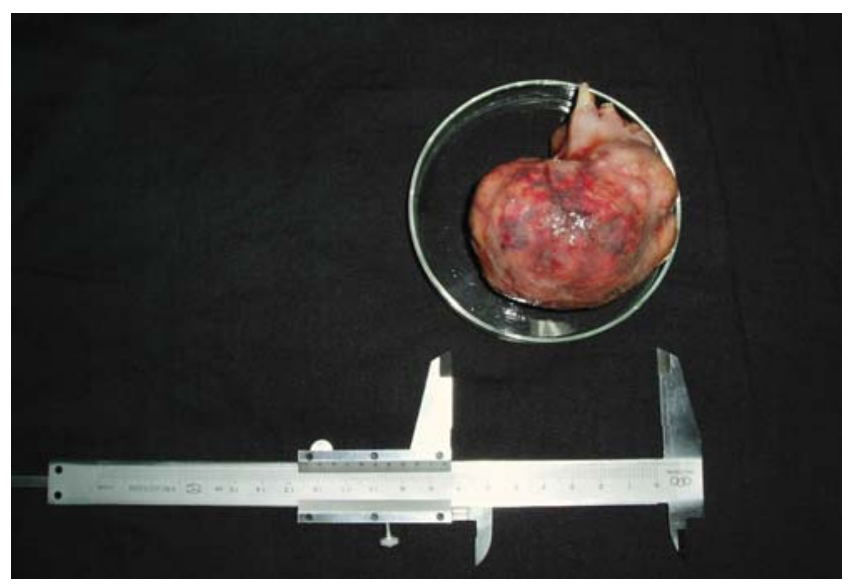

Figure 3. Orthotopically implanted tumor demonstrated expansive growth, affected the neighbouring organs and demonstrated vascularized property.

Tumor volume growth curve. One week after injection, all rats from Groups D and E developed a solitary nodule. The tumors expanded vigorously with time-lapse, especially in the first 3 weeks. Then, the tumor-bearing rats presented dyscrasia and exhaustion. Ultrasound demonstrated a well-demarcated low echo mass. Tumor size was measured and calculated weekly post-inoculation. At 24 days post-inoculation, the first spontaneous death occurred, which was from Group D, with a 


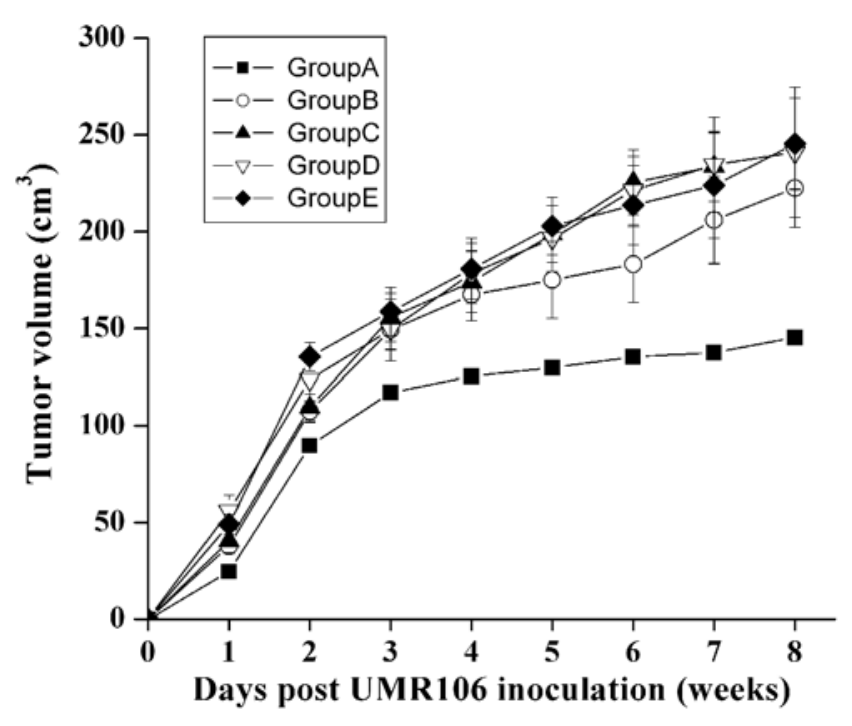

Figure 4. Time-course of orthotopically implanted osteosarcma growth. Tumor size was measured and calculated weekly post-inoculation. Data represent mean volume \pm SEM (only tumor-bearing rats were counted).

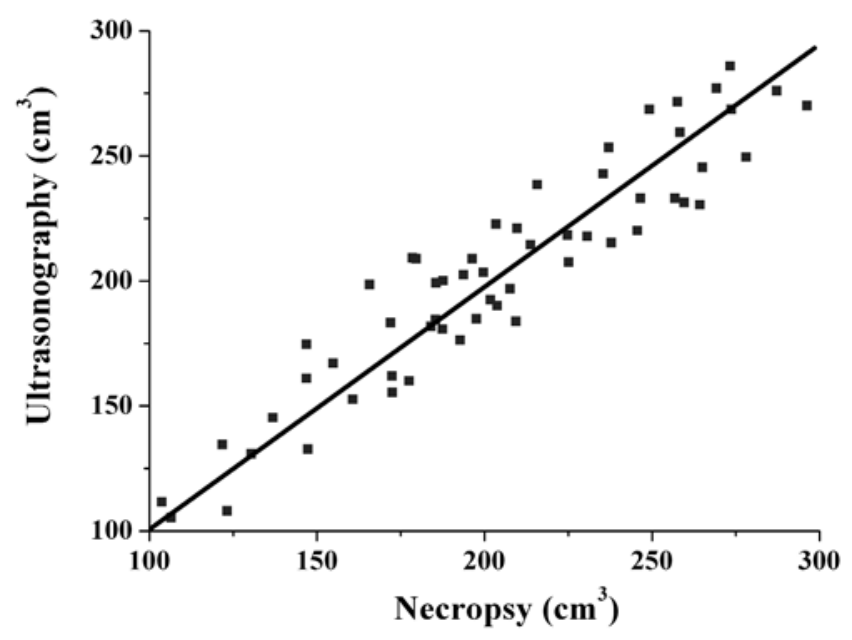

Figure 5. Simple linear regression test showing positive correlation between ultrasound and necropsy findings for tumor volume $\left(\mathrm{cm}^{3}\right)\left(\mathrm{R}^{2}=0.887 ; \mathrm{p}<0.01\right)$.

considerable tumor volume of $259.4 \mathrm{~mm}^{3}$ (ultrasonography). Ultrasound demonstrated a hypervascular property of the tumor accompanied with a central necrosis.

While in Group A, there was only one rat inoculated with visible nodule, tumor volume reaching $145.3 \mathrm{~cm}^{3}$ at the death phase (52 days after inoculation). Of the remaining 19 , local nodules were not observed, neither distant metastases detected in the lungs, as opposed to that commonly observed with malignant bone cancer in rats or humans. Four and twelve animals $(66 \%)$ presented regional tumor dissemination in Group B and C, respectively, either towards abdominal cavity or pelvic cavity.

On the whole, all of the tumor-bearing animals died within eight weeks; the median survival time was $36.28 \pm 5.76$ days (Fig. 4). In almost all cases, the tumor occupied most of the lungs with large areas of necrosis and hemorrhagic foci.
Analysis of the correlation between ultrasound and necropsy findings. On the spontaneous death day, each inoculated rat with visible nodules was submitted to Doppler ultrasonography to acquire the various tumor diameters and obtain the volume as previous formula indicated. Then the tumor-bearing rat was necropsied and implantated tumor was removed for histological measurement by sliding caliper. There were a total of 57 rats in this comparison process. Simple linear regression test demonstrated the correlation between findings of tumor volume $\left(\mathrm{cm}^{3}\right)$ obtained with ultrasound and necropsy was positive $(\mathrm{r}=0.942 ; \mathrm{p}<0.01)$ (Fig. 5). The necropsy confirmed ultrasonography findings with regard to tumor volume and location.

Histopathological evaluation. All of the animals were submitted to necropsy after spontaneous death in consequence of the neoplasm. Histopathologically, UMR106 cell line was a poorly differentiated osteosarcoma, well-demarcated with surrounding tissue. In almost all cases, pathological tissue occupied most of the lumps in the leg with large areas of necrosis and hemorrhagic foci. Microscopically, the implanted tumor was characterized by fusiform or triangle cells along nutrient vessels with nuclear pleomorphism, mitoses, bone invasion and necrosis (Fig. 6).

Most orthotopically implanted tumors formed a peribronchiolar mass with adjacent lung showing signs of swelling and congestion. Microscopically, the pulmonary alveolus walls were affected by the neoplasm, with loss of their normal characteristics (Fig. 7).

\section{Discussion}

The use of laboratory animal models is an essential component of preclinical cancer therapy investigations. Implantation of allogenic tumors are most commonly modeled in nude mice, whose small size is convenient for most experimental applications but limits the investigation of more complex surgical procedures. For these kinds of studies, the nude rat is more appropriate (15). The nude rat has an autosomal recessive mutation which results in the lack of a functional thymus. T lymphocytes and cell-mediated immunity are markedly reduced and graft rejection is diminished or delayed. However, the primary disadvantage of the nude rat is its relative immune competence compared to athymic nude mice. If researchers were further to explore the immunoprotection or therapeutical effect of immune system, nude rats do not work. The rat (SD or Wistar) plays a very important role in the bone field as well, especially in the evaluation of the immune system. Scientists are accustomed to select rat as model animal for bone primary affection or metastases mainly because of the size of the rat bone allows a better handling and the interest in evaluating biomaterials or therapeutic molecules targeting cancer in order to reduce fractures.

UMR106 is an aggressive, poorly immunogenic osteosarcoma cell line, which has strong carcinogenic capability and a high lung metastasis frequency. It was originally derived from a rat transplantable osteogenic sarcoma with an osteoblastic phenotype. In 2001, UMR106 cells were first inoculated into the tibia of athymic mice by Fisher et al (16). Inoculated osteosarcoma development was associated with 


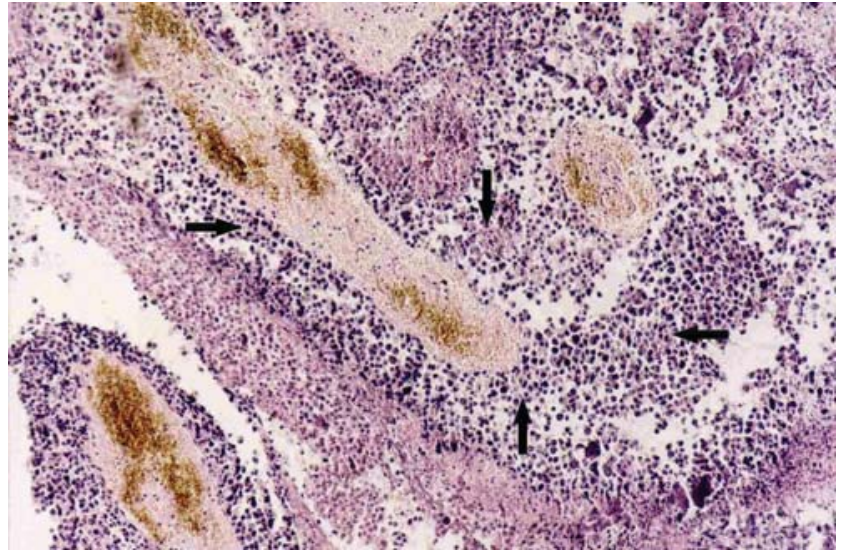

Figure 6. Histopathological test with hematoxylin-eosin staining demonstrated an implanted osteosarcoma (x100). Implanted tumor was characterized by fusiform or triangle cells (arrow) along nutrient vessels with nuclear pleomorphism, mitoses, bone invasion and necrosis.

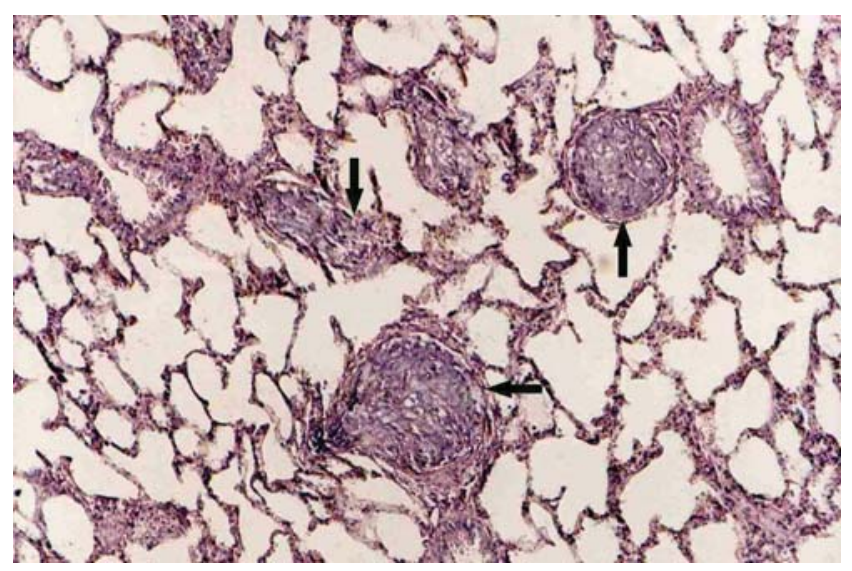

Figure 7. Histopathological appearance of lung metastasis nodules (x40) Orthotopic implanted tumor on Group D formed a peribronchiolar mass (arrow) with adjacent lung showing signs of swelling and congestion. The pulmonary alveolus walls were affected by the neoplasm, with loss of their normal characteristics.

tibial swelling and lameness, and radiographic changes included osteolysis and new bone formation. Lung metastases developed spontaneously. In a previous study, we used the subcutaneous injection technique to establish tumor-bearing model, the requirement for implanted cell amount was considerable (at least at the level of $10^{7}$ ). Even worse, the reproducible stability was not acceptable. If the rats were $>3$ weeks old, the oncogenic rate was uncertain. Others have also questioned the subcutaneous inoculation method. Tumor biology in subcutaneous models remained questionable and unreliable, and orthotopic models are now the standard (1721).

Cancer development depends upon changes in the heterotypic interactions between incipient tumor cells and their normal neighbors. Moreover, once formed, virtually all types of tumors, including their metastatic outgrowths, continue to harbor complex mixtures of several cell types that collaborate to create malignancy (22). The microenvironment of medullary cavity is more suitable than subcutaneous tissue in imitating human osteosarcoma surroundings, which provides essential conditions for the nodule growth, even though the detailed factors have not been identified. As a result, in the orthotopically implanted tumor it is much easier to present vigorous expanding, invasion and metastasis, which demonstrates the microenvironment of target organs acting in inoculated tumor cell progression. For orthotopic implantation, needle injection is simple and in principle is suited to inoculate a calculated number of tumor cells that is critical for controlling tumor growth and size. The results showed, tumor take rate was $100 \%$ for implants with $8 \times 10^{5}$ tumor cells or more, which was much less than the amount required for subcutaneous implantation. Additionally, orthotopic implantation technique reduced the requirement for target animals' age. In this study, 5-week-old rats were also available for inoculation, which resulted in the improvement of animal resistance to circumstance changes or surgical intervention. In the whole experiment process, surgical mortality was $0 \%$.

The purpose of these experiments was to establish a reliable and representative animal model for osteosarcoma, consolidate the work of previous researchers and our earlier studies. In the process of trying to establish this orthotopic implantation method, we observed highly variable tumor take rates and tumor growth properties. Preliminary experiments showed observable nodules began to emerge from the groups inoculated at the level of $10^{5}$. Based on the experience, we set five arithmetic groups, with inoculation cell amounts from $2 \times 10^{5}$ to $1 \times 10^{6}$. The results showed, in groups $\mathrm{D}$ and $\mathrm{E}$, tumor cells turned into nodules in $100 \%$ of the animals, as documented by ultrasound within one week following intraosseous implantation with $8 \times 10^{5}$ cells or more. If the amount of implanted cells were not enough, tumor formation rate was unreliable. From the results obtained in the present and previous studies, we can infer that the present rat osteosarcoma model was feasible: the take rate was high, surgical mortality was negligible and the procedure was simple to perform and easily reproduced. Studies on immunotherapy of rat osteosarcoma based on subcutaneous inoculation models have been published $(12,23)$ and we are currently using this improved model to assess tumor responsiveness to allogenic tumor vaccine, a novel immunotherapeutic agent able to elevate MHC antigens and costimulatory molecule presentation.

The most used technique for the detection of bone tumor is X-ray examination. However, the measurement of radiological tumor size does not account for the amount of necrosis (a characteristic of most malignant bone tumor) nor is there any true consideration of the amount of surrounding vasogenic oedema, making direct radiological measurements of questionable quantitative utility. MRI seemed to have considerable predominance to overcome these disadvantages and could act as the standard technique for in vivo imaging of these tumors. However, the time and cost aspect of animal MRI limits most research and the interpretation of data is limited by the resolution of the magnet. Doppler ultrasound has been shown to be of such high sensitivity to contrast agents that even a single adventitia echo can be detected. Besides, ultrasound is such a convenient and harmless technique, that increasing number of physicians are selecting to use it for tumor diagnosis, localization and measurement 
(24-28). In this study, ultrasound was also demonstrated as a highly accurate tool to visualize and measure cancer every week, giving high correlation between the average ultrasonography and histology of the tumor volume.

In summary, we have reported a method of producing rat osteosarcoma in the median or distal femoral shaft of the immunocompetent rat, using UMR106 osteosarcoma cell line, which yields excellent tumor take rates and consistent, reliable growth patterns. This technique is an ideal means of tumor model establishment also owing to its easier manipulation, shorter operating time, less blood loss, quicker postoperative recovery and high survival of experimental animals. The UMR106 cell line has strong carcinogenic capability and high lung metastasis frequency. The previous and present studies both showed, that when the amount of implants was sufficient, the technique of inoculation was suitable, this cell line was reliable for tumor model establishment. Simple linear regression test demonstrated the correlation between findings of tumor volume obtained with ultrasound and necropsy was positive. Based on the above result, we conclude that, ultrasound is a highly accurate tool for tumor diagnosis, localization and measurement and may be recommended for monitoring tumor growth in further studies.

\section{Acknowledgements}

This study is sponsored by the National Natural Science Foundation (30330610, CHN). We would like to thank Professor Qiu Xiuchun for his technical help and Zhao Hong for his efforts in taking care of animals. We also would like to thank Dr Zhang Yunfei for his valuable advice.

\section{References}

1. Fan QY, Ma BA, Zhou Y, Zhang MH and Hao XB: Bone tumors of the extremities or pelvis treated by microwaveinduced hyperthermia. Clin Orthop 406: 165-175, 2003

2. Blouin S, Baslé MF and Chappard D: Rat models of bone metastases. Clin Exp Metastasis 22: 605-614, 2005.

3. Taïeb D, Hassad R, Sebag F, Colavolpe C, Guedj E, Hindié E, Henry JF and Mundler O: Tomoscintigraphy improves the determination of the embryologic origin of parathyroid adenomas, especially in apparently inferior glands: imaging features and surgical implications. J Nucl Med Technol 35: 135$139,2007$.

4. Kurth AA and Müller R: The effect of an osteolytic tumor on the three-dimensional trabecular bone morphology in an animal model. Skeletal Radiol 30: 94-98, 2001.

5. Gomes Neto A, Simão AF, Miranda Sde P, Mourão LT, Bezerra NP, Almeida PR and Ribeiro Rde A: Experimental rat lung tumor model with intrabronchial tumor cell implantation. Acta Cir Bras 23: 84-92, 2008.

6. Dickson GR, Geddis C, Fazzalari N, Marsh D and Parkinson I: Microcomputed tomography imaging in a rat model of delayed union/non-union fracture. J Orthop Res 26: 729-736, 2006.

7. Chen F, Sun X, De Keyzer F, Yu J, Peeters R, Coudyzer W, Vandecaveye V, Landuyt W, Bosmans H, Van Hecke P, Marchal G and Ni Y: Liver tumor model with implanted rhabdomyosarcoma in rats: MR imaging, microangiography, and histopathologic analysis. Radiology 239: 554-562, 2006.

8. Pan MH, Huang SC, Liao YP, Schaue D, Wang CC, Stout DB, Barrio JR and McBride WH: FLT-PET imaging of radiation responses in murine tumors. Mol Imaging Biol 10: 325-334, 2008.
9. Braun RD and Vistisen KS: Measurement of human choroidal melanoma xenograft volume in rats using high-frequency ultrasound. Invest Ophthalmol Vis Sci 49: 16-22, 2008.

10. Stieger SM, Bloch SH, Foreman O, Wisner ER, Ferrara KW and Dayton PA: Ultrasound assessment of angiogenesis in a matrigel model in rats. Ultrasound Med Biol 32: 673-681, 2006.

11. Satoh H, Morimoto Y, Arai T, Asanuma H, Kawauchi S, Seguchi K, Kikuchi M and Murai M: Intravesical ultrasonography for tumor staging in an orthotopically implanted rat model of bladder cancer. J Urol 177: 1169-1173, 2007.

12. Yu Z, Ma B, Zhou Y, Zhang M, Long H, Wang Y and Fan Q: Allogeneic tumor vaccine produced by electrofusion between osteosarcoma cell line and dendritic cells in the induction of antitumor immunity. Cancer Invest 25: 535-541, 2007.

13. Bryant MJ, Chuah TL, Luff J, Lavin MF and Walker DG: A novel rat model for glioblastoma multiforme using a bioluminescent F98 cell line. J Clin Neurosci 15: 545-551, 2008.

14. Carlsson G, Gullberg B and Hafström L: Estimation of liver tumor volume using different formulas - an experimental study in rats. J Cancer Res Clin Oncol 105: 20-23, 1983.

15. Strojnik T, Kavalar R and Lah TT: Experimental model and immunohistochemical analyses of U87 human glioblastoma cell xenografts in immunosuppressed rat brains. Anticancer Res 26: 2887-2900, 2006

16. Fisher JL, Mackie PS, Howard ML, Zhou H and Choong PF: The expression of the urokinase plasminogen activator system in metastatic murine osteosarcoma: An in vivo mouse model. Clin Cancer Res 7: 1654-1660, 2001.

17. Okubo H, Takei Y, Serizawa N, Enomoto N, Ikejima K and Sato N: Orthotopic hepatocellular carcinoma model with a controlled and reproducible tumorigenicity. J Gastroenterol Hepatol 22: 423-428, 2007.

18. Sho A, Kondo S, Kamitani H, Otake M and Watanabe T: Establishment of experimental glioma models at the intrinsic brainstem region of the rats. Neurol Res 29: 36-42, 2007.

19. Gelbard A, Kupferman ME, Jasser SA, Chen W, El-Naggar AK, Myers JN and Hanna EY: An orthotopic murine model of sinonasal malignancy. Clin Cancer Res 14: 7348-7357, 2008.

20. Shi J, Wei PK, Zhang S, Qin ZF, Li J, Sun DZ, Xiao Y, Yu ZH, Lin HM, Zheng GJ, Su XM, Chen YL, Liu YF and Xu L: OB glue paste technique for establishing nude mouse human gastric cancer orthotopic transplantation models. World J Gastroenterol 14: 4800-4804, 2008

21. Horiguchi Y, Kikuchi E, Ozu C, Nishiyama T, Oyama M, Horinaga M, Yoshioka K and Tachibana M: Establishment of orthotopic mouse superficial bladder tumor model for studies on intravesical treatments. Hum Cell 21: 57-63, 2008.

22. Hanahan D and Weinberg RA: The hallmarks of cancer. Cell 100: 57-70, 2000.

23. Yu Z, Fan Q, Hao X and Long H: Specific antitumor effects of tumor vaccine produced by electrofusion between osteosarcoma cell and dendritic cell in rats. Cell Mol Immunol 1: 454-460, 2004.

24. Abboud B, Sleilaty G, Rabaa L, Daher R, Abou Zeid H, Jabbour H, Hachem K and Smayra T: Ultrasonography: highly accuracy technique for preoperative localization of parathyroid adenoma. Laryngoscope 118: 1574-1578, 2008.

25. Zhu Q, Tannenbaum S and Kurtzman SH: Optical tomography with ultrasound localization for breast cancer diagnosis and treatment monitoring. Surg Oncol Clin N Am 16: 307-321, 2007.

26. Bolintineanu S, Brad S, Brad V and Motoc A: The value of radioimaging methods in the diagnosis of chest wall metastases. Rom J Morphol Embryol 47: 79-81, 2006.

27. Mesurolle B, El-Khoury M, Hori D, Phancao JP, Kary S, Kao E and Fleiszer D: Sonography of postexcision specimens of nonpalpable breast lesions: value, limitations, and description of a method. AJR Am J Roentgenol 186: 1014-1024, 2006.

28. Kuo YL, Yao WJ and Chiu HY: Role of sonography in the preoperative assessment of neurilemmoma. J Clin Ultrasound 33: 87-89, 2005. 